\title{
Flow Field Analysis of Different Intake Bump (Compression Surface) Configurations on a Supersonic Aircraft
}

\author{
I. Arif, M. Ahmed, S. Salamat and M. Ghafoor
}

\begin{abstract}
Aircraft intake plays a vital role in overall performance of aircraft. It must be able to provide required mass flow of air to engine compressor and should be able to maintain maximum possible pressure recovery at different flight conditions. Even a slight reduction in pressure recovery can result in declined engine thrust. Diverterless supersonic inlet (DSI) has gained significant importance in aircraft intake design due to its favorable characteristics such as weight reduction, pressure recovery and less flow distortion as compared to other conventional intakes. These intakes have specific bump surfaces ahead of intake duct which diverts the boundary layer and ensures smooth flow inside duct. In this paper different bump surfaces are modeled and analyzed to evaluate their performance. The research is mainly divided into two different parts. Initially, 04 different bump configurations are modeled in MATALB ${ }^{\circledR}$ based on their maximum amplitude, location of maximum amplitude, and initial / final bump angles. Bump No 1 has smooth initial angle but blunt rear angle; Bump No 2 has similar angle to that of Bump No 1 but has a lower amplitude; Bump No 3 has a smooth initial and smooth rear angle; whereas Bump No 4 has blunt initial and blunt rear angle. The outer perimeter of each bump geometry is kept consistent with bump geometry on fighter aircraft under study. All the geometries are meshed using unstructured tetrahedral meshing scheme for numerical analysis in ANSYS FLUENT ®. Analysis is carried out at different flow conditions (Mach No and angles of attack). Different flow features such as flow distortion, boundary layer diversion, vortices and pressure recovery are analyzed for each bump for comparative analysis. Finally, the modeled bumps are integrated on fighter aircraft to analyze the intake behavior and carry out a comparative analysis with existing aircraft Diverterless Supersonic Intake (DSI) performance. The analysis revealed that at subsonic regime, Bump No 1 and 2 resulted in similar pressure recovery as that of aircraft DSI intake. However, a significant variation in pressure recovery was observed at supersonic speeds. Bump No 1 pressure recovery was observed to be much higher than Bump No 4 at all conditions. Also, with same bump angles, higher amplitude bump resulted in much better flow behavior and higher pressure recovery than lower amplitude bump. The research helped in understanding the effect of bump geometry on overall intake performance and can be utilized for different aircraft.
\end{abstract}

Index Terms-Bump, Intake, Performance, Pressure Recovery

Manuscript received May 5, 2017.

I. Arif is an Assistant Professor with the National Institute of Science and Technology, Islamabad (e-mail: arsalan_sayani@ cae.nust.edu.pk).

M. Ahmed is an Assistant Professor with the National Institute of Science and Technology, Islamabad (e-mail: mahmed@ cae.nust.edu.pk).

S. Salamat is an Associate Professor with the National Institute of Science and Technology, Islamabad (e-mail: ssalamat@ cae.nust.edu.pk).

M. Ghafoor is a Graduate of National Institute of Science and Technology, Islamabad (e-mail: mudsr1995@gmail.com@cae.nust.edu.pk).

\section{INTRODUCTION}

Diverterless supersonic inlet (DSI) has gained significant importance in aircraft intake design since initial NASA research in 1950s due to its favorable characteristics as compared to other conventional intakes [1]. It adds higher stealth characteristics to the inlet [2] and helps in reduction of aircraft weight by replacing its inlet mechanism [3,4,5].Also, Diverter less Supersonic Inlet (DSI) provides favorable mass flow rates as compared to clean intake [6]. The parent theory behind the design of bump is based on Waverider concept [7].

Presently Diverter less Supersonic Inlet (DSI) is successfully integrated on few developed supersonic aircraft. This research work on bump configurations presents how the flow is redirected after passing over contoured bump. The research is based on numerical analysis of flow behavior over different bump configurations and a comparative study among different configurations. The research is mainly divided into two different parts. Initially, 04 different bump configurations are modeled in MATALB $\AA$ based on their maximum amplitude, location of maximum amplitude, and initial / final bump angles. Finally, the modeled bumps are integrated on fighter aircraft to analyze the intake behavior and carry out a comparative analysis with existing aircraft Diverterless Supersonic Intake (DSI) performance. Zero Angle of attack (AOA) and sideslip angle is maintained zero for all simulations.

\section{MODELLING OF BUMPS}

Geometry of the bump is defined by longitudinal curves at different stations [6]. Cone flow has the flow relieving effect due to its 3D nature. So when it is compared to a wedge it can be observed that due to this flow relieving effect for same body angle and same Mach, the shock wave which forms in cone is weaker. Therefore, surfaces same as cone flow describes the design principle for bump geometry to achieve desired effects $[8,9]$ as shown in Fig 1. Hyperbolic approximation of cone flow streamlines is used to design the bump. 


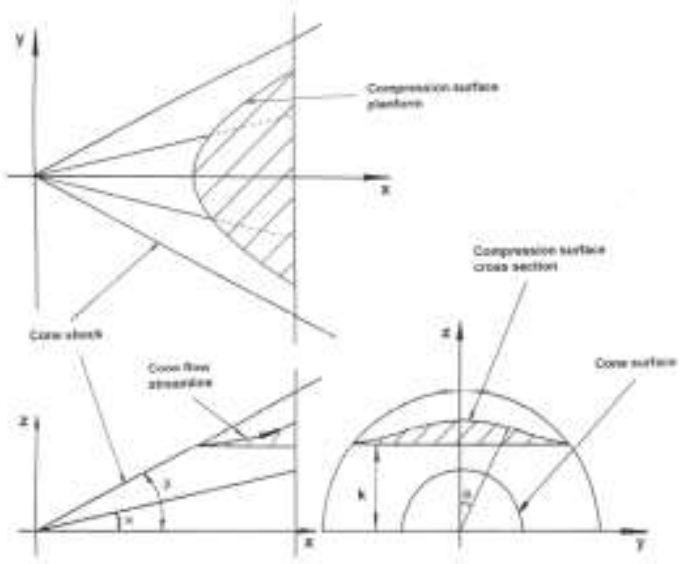

Fig. 1.Typical Bump Geometry (Planar Views)

During this research, mathematical equations for intake bumps [10] were implemented in MATLAB ${ }^{\circledR}$. The mathematical equations used for modeling of bump geometries are as follows:

$$
\begin{gathered}
z=K=\text { const } \\
\tan \beta=\frac{Z w \sec \theta}{x}
\end{gathered}
$$

The equation for the intersection of the plane and shock surface was:

$$
x^{2} \tan ^{2} \beta-y^{2}=K^{2}
$$

The equation for the streamline was:

$$
r^{2}=z^{2} \sec ^{2} \theta=x^{2} \tan ^{2} \alpha+c
$$

The streamline equation was solved for the $\mathrm{z}$-coordinate before implementing it in MATLAB $\AA$ to create the bump-geometry. Here $\alpha$ is the cone angle and $\mathrm{c}$ is a constant, the equations we get are:

$$
z=\sqrt{\frac{x^{2} \tan ^{2} \alpha+c}{\sec ^{2} \theta}}=\frac{\sqrt{x^{2} \tan ^{2} \alpha+c}}{\left(\frac{1}{\cos \alpha\left(\tan ^{-1}\left(\frac{1}{K}\right)\right)}\right)^{2}} \times A \times B
$$

The initial geometry had two imperfections that were corrected as follows; first the discontinuous boundaries along the side edges were corrected by multiplying the function $\mathrm{z}$ with a trigonometric function A. Second, the boundary at the end downstream was not converging. To correct this problem, the function $\mathrm{z}$ was also multiplied with the function $\mathrm{B}[6,10]$.

$$
\begin{gathered}
A=\sin (x) \\
B=\sin \left(\frac{y+\pi}{2}\right)
\end{gathered}
$$

By using the above mentioned equations, 3D surfaces were generated in MATLAB $囚$. The MATLAB generated geometries were not compatible with CAD software. To make these surfaces compatible with a CAD software, points of these surfaces were obtained from MATLAB ${ }^{\circledR}$ and were plotted in MS Excel®. The data is further linked to CATIA for CAD modeling. The bumps were named as Config 1, 2, 3 and 4 based on their geometric features. The final measurements of all bump geometries and max amplitude of each bump is shown in Table 1. The generated 3D surfaces of four different bumps in MATLAB ${ }^{\circledR}$ are shown below in Fig 2.

TABLE I:

BUMP FEATURES AND DIMENSIONS

\begin{tabular}{|l|c|c|c|c|}
\hline FEATURES & CONFIG 1 & CONFIG 2 & CONFIG 3 & CONFIG 4 \\
\hline START & SOFT & SOFT & SOFT & SHARP \\
\hline END & SHARP & SHARP & SOFT & SHARP \\
\hline $\begin{array}{l}\text { MAX } \\
\text { AMPLITUDE } \\
(\mathrm{m})\end{array}$ & 0.20 & 0.10 & 0.20 & 0.20 \\
\hline LENGTH (m) & 1.4 & 1.4 & 1.4 & 1.4 \\
\hline WIDTH (m) & 0.775 & 0.775 & 0.775 & 0.775 \\
\hline
\end{tabular}
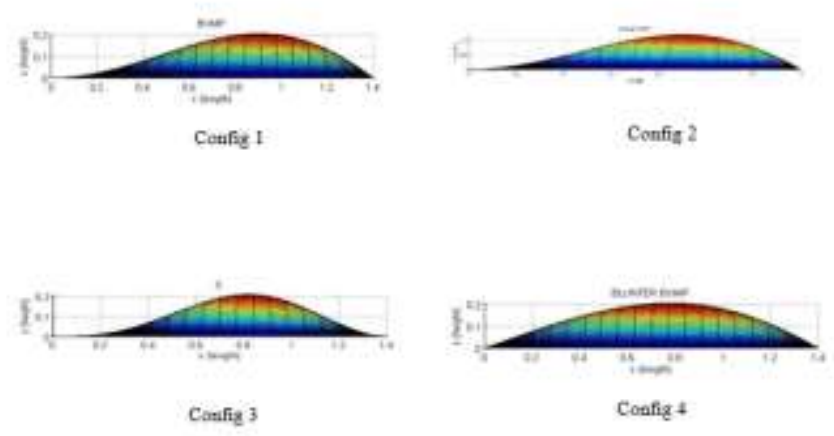

Fig. 2 Modeled Bump Geometries in MATLAB®

\section{COMPUTATIONAL SETUP}

For numerical analysis of bump geometries, modeled geometries were imported in ANSYS® ICEM for mesh generation. This step was followed by numerical simulations in Fluent ${ }^{\circledR}$ software. Unstructured meshing scheme was used for this analysis. Square domain was made around the bump and the bottom surface was designated as wall to duplicate the effect of fuselage. Analysis is carried out at different flow conditions (Mach No and angles of attack). Simulations were carried out at zero side slip angle and zero angle of attack. Different flow features such as flow distortion, boundary layer diversion, vortices and pressure recovery are analyzed for each bump for comparative analysis. The calculated pressure recovery was later compared with actual aircraft intake pressure recovery to validate and compare the results.

\section{A. Grid Generation}

Grid generation is one of the key steps in numerical simulation. It is imperative to design and generate a smart grid which is dense and fine enough to capture all flow phenomena accurately. However, a very fine mesh can be computationally expensive [11]. Hence, for this research, a smart grid was generated which is very fine at important areas such as bump surface, upstream and downstream of bump. Domain size was kept 20 times the length of bump for accurate analysis. Much 
finer mesh is made near the bump surface as compared to domain surfaces for better results. For surface meshing, all tri mesh technique with Patch independent technique is used for this analysis. Turbulent Law $\mathrm{y}^{+}$values were kept at optimum level for subsonic and supersonic speeds [12]. A size function was also applied to gradually coarsen the mesh size away from the bump surface. Similar mesh size was kept for all bump geometries and intake duct to maintain consistency in numerical analysis. A mesh size of 3.2 million was selected after performing a thorough grid independence test between three mesh sizes of 2.4 million, 3.2 million and 5 million. The final selected volume mesh is shown in Fig. 4.

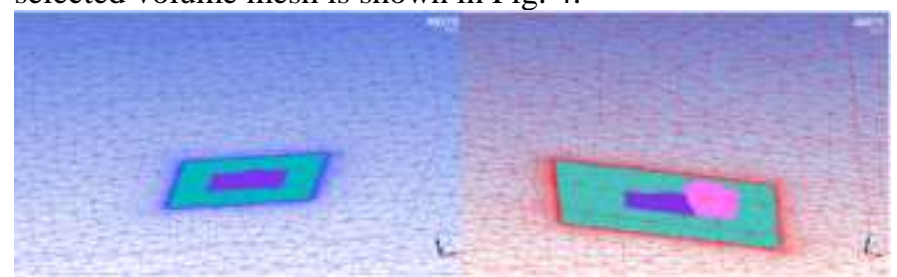

Fig. 4. Meshed geometry of smooth bump without intake (left) and with intake (right)

\section{B. Analysis Strategy}

Numerical analysis is based on Reynolds-Averaged Navier-Stokes (RANS) equations to predict the flow behavior with time dependency. Fluent 16.0 was used for analysis and to visualize flow properties. Density based solver was chosen for present research and explicit technique was implemented in Fluent ${ }^{\circledR}$ [13]. Flow discretization was selected as 2 nd order upwind, whereas 1st order upwind scheme was used for turbulent viscosity. Relaxation parameters were set in accordance with solution stability behavior for better solution stability and a courant number of 1 was set.

\section{Boundary Conditions}

Bump surfaces were selected as walls. Pressure far field boundary condition was selected for domain except for bottom surface which was taken as wall to simulate the fuselage wall. All flow conditions were calculated at static sea level conditions and atmospheric data was taken accordingly. For bump analysis with intake duct, the duct wall was taken as no slip wall.

\section{Turbulence Model}

Unfortunately there is no single model which is universally acceptable for all the cases. One must keep in mind the pros and cons of the model he is using. For present study, Spalart-Allmaras (SA) one equation model was used as it quite is feasible for typical aerospace applications [14] involving boundary layer phenomenon. Also it demands less computational cost because it allows somewhat coarser mesh near the solid wall. A single solution took an average time of two days to stabilize with multi-processor (Eight Core processor) high end workstation.

\section{RESUlTS AND DiSCUSSION}

In the first part of this research, analysis for bump surfaces without intake duct was carried out at two different Mach Numbers, 0.6 and 1.2. Flow behavior was analyzed qualitatively and compared for all four bump configurations. Qualitative analysis revealed how the flow is redirected and which configuration gave better results. After this analysis, intake duct was integrated with all configurations and pressure recovery was calculated. It must be kept in mind at this time that bump with better flow behavior may or may not give same results after integration of intake duct. Therefore, in the second part of the research, bump surfaces were integrated with intake duct for analysis. Pressure recovery was calculated for each bump geometry and comparative analysis was carried out with existing supersonic aircraft data [15].

\section{A. Analysis of Bump Surfaces without Intake Duct}

Surface flow behavior is analyzed for all simulations to study the effectiveness of bump geometries. Streamlines are generated prior to bump surface to observe how well the bumps redirect the flow or either they generate swirls or not. Flow separation and vortices which are formed downstream of bumps are also critically observed. Surface flow at Mach No 0.6 is shown in Fig 5. for all four geometries.

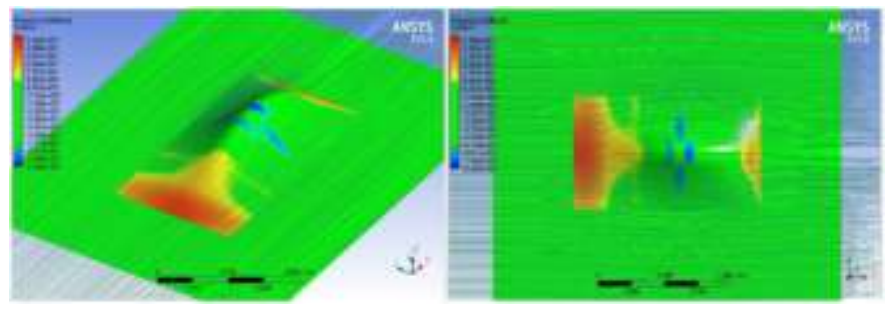

Fig. 5a. Streamlines (Pressure) over Config 1 at Mach No. 0.6

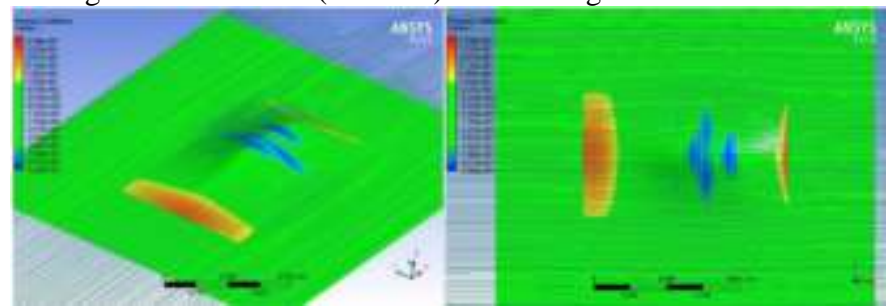

Fig. 5b. Streamlines (Pressure) over Config 2 at Mach No. 0.6

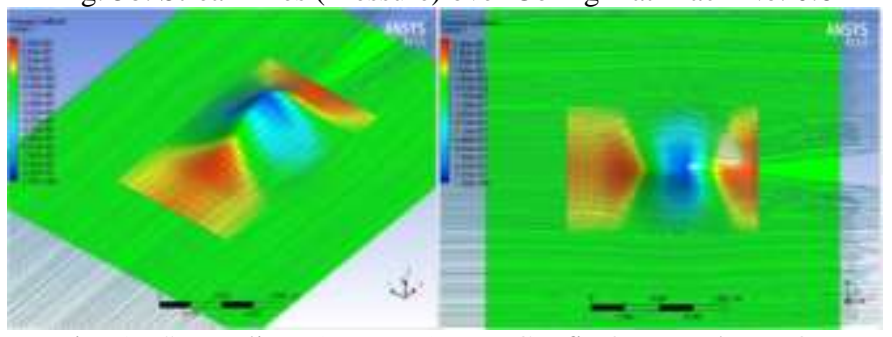

Fig. 5c. Streamlines (Pressure) over Config 3 at Mach No. 0.6

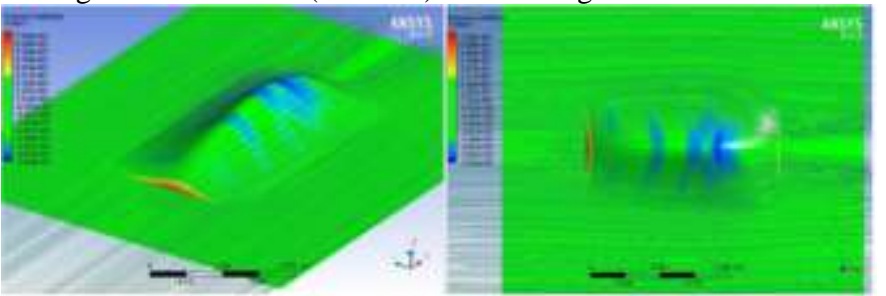

Fig. 5d. Streamlines (Pressure) over Config 4 at Mach No. 0.6

The color on the surfaces indicate different values of Coefficient of Pressure ( $\mathrm{Cp}$ ) which indicates the pressure acting on the surface, where red areas indicating high pressure region and blue area indicating lower pressure region. In areas where 
the pressure is low, there is a tendency of flow separation and more turbulence. Comparative analysis of bump geometries reveal that Smooth Bump produces less swirls and almost no flow separation whereas Soft Bump gives rise to maximum swirls. Soft bump has prominent low pressure areas therefore its separation and magnitude of turbulence is also more as compared to other geometries. Blunt bump also produces swirls but not as severe as soft bump. The small bump is redirecting the flow in a best way and no separation is observed at valley of this bump. Bump smooth and smaller produces few low pressure areas at subsonic speeds. Due to the reason that smooth and small bump had high pressure areas at start and also at end, they show very little or no turbulence at all.

At supersonic speed of Mach No 1.2, the shock effects also come into play and due to these compression effects, severe swirls rise. Here we can see that magnitude of highest pressure is increased which is favorable. At the same time we can see that at the rear end there is low pressure area which may result in the turbulence which is undesirable. Surface flow at Mach No 1.2 is shown in Fig 6. for all four geometries.

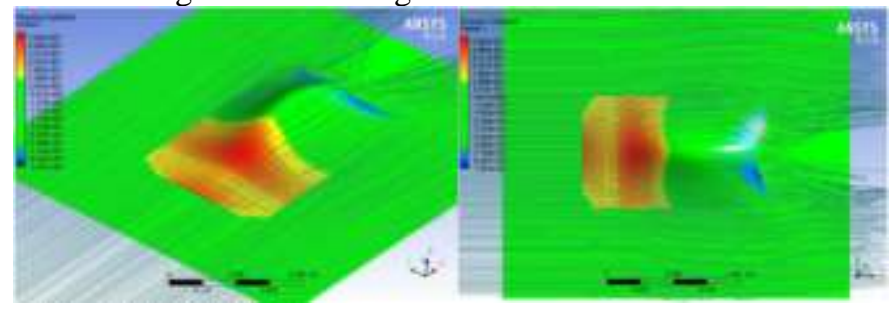

Fig. 6a. Streamlines (Pressure) over Config 1 at Mach No. 1.2

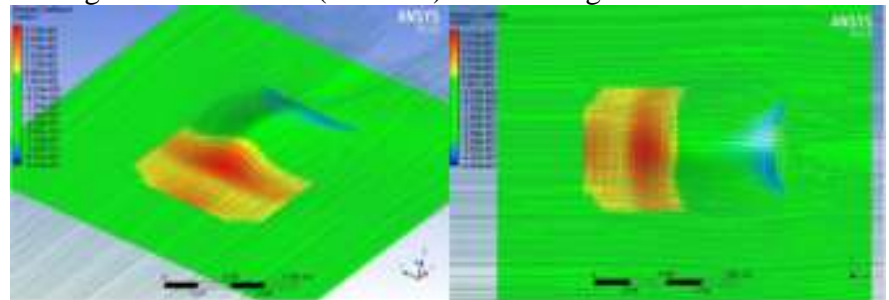

Fig. 6b. Streamlines (Pressure) over Config 2 at Mach No. 1.2

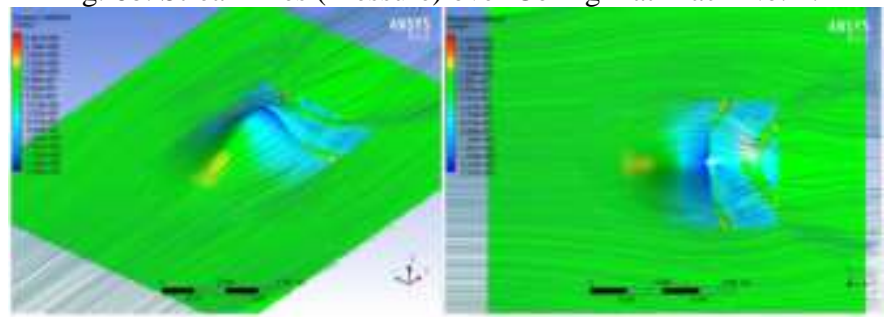

Fig. 6c. Streamlines (Pressure) over Config 3 at Mach No. 1.2

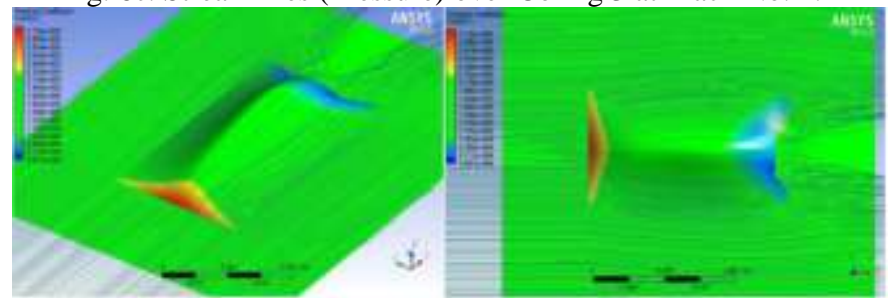

Fig. 6d. Streamlines (Pressure) over Config 4 at Mach No. 1.2

At supersonic speed, for all bump geometries two counter rotating vortices are formed at the bump valley. These vortices are due to the presence of low pressure areas at these locations. Flow over contoured bump is attracted at low pressure areas which form these counter rotating vortices. It can be seen that the flow is separating at the rear of Config 1 and swirls are also produced which results in turbulence at the rear end. Streamlines over Config4 shows that it creates more swirls than the Config 1 and produces higher turbulence. At the start of its surface, high pressure is observed but when we go along the flow direction the pressure decreases and at the end of bump surface it slightly increases but the increase is insufficient to keep the flow attached to the surface.

At supersonic speed Config3 shows sever swirls and very high turbulence. Here we can see that there are few high pressure areas. Due to low pressure areas the streamlines are not attached to the surface which results in the phenomenon of separation. Hence, Config3 shows highest turbulence. At subsonic speed, turbulence of Config 2 is almost negligible. Even at supersonic speed it is producing very less turbulence. One of the governing reasons is its magnitude, which is not too big to give rise to swirls so the flow remains streamlined.

\section{B. Analysis of Bump Surfaces with Intake Duct}

For analysis of bump surfaces with intake duct, pressure contours are analyzed critically. These pressure contours are drawn at the cut plane through center of all bumps. The use of contour plot is usually not targeted for precision evaluation of the numerical values between contour lines. It is aimed to only visualize the flow and its behavior when it passes through intake duct. At the start of bump surface due to compression, the pressure increases which is indicated by red area representing high pressure location. All cases at Mach No 0.6 can be seen below in Fig. 7:

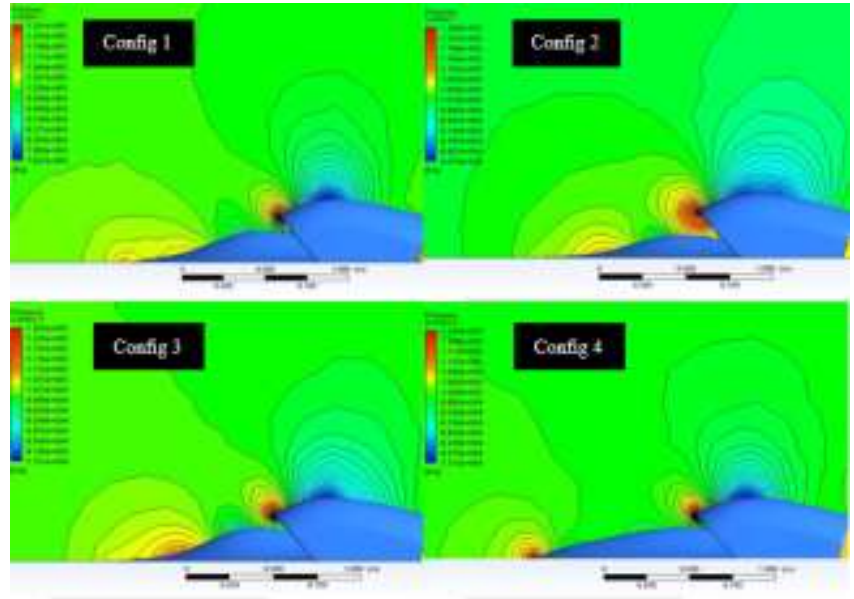

Fig. 7. Pressure Contours over bump configurations at Mach No. 0.6

Velocity downstream of intake duct is also calculated. At subsonic Mach No 0.6, there are much less areas with high pressure or compression. Config 4 is showing higher compression at start, which is due to the reason that it has greater body angle as compared to other bumps. At the cowl lip compression can be seen clearly. Config 3 is showing negligible compression due to its smaller amplitude.

At Mach 0.95 the compression is greater as compared to Mach 0.6 but still there are no prominent shock waves at this speed. At Mach 0.95 all configurations can be seen below in the Fig 8: 


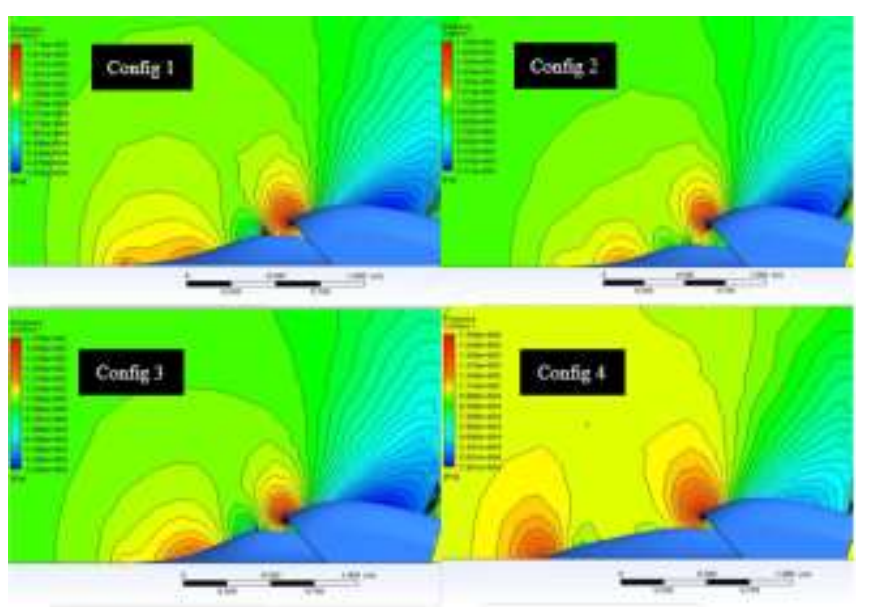

Fig. 8. Pressure Contours over bump configurations at Mach No. 0.95

Compression at Mach No 0.95 is much lesser for Config 1 and Config 2. As it is described earlier as well that Config 3 and 4produces higher turbulence and flow is not redirecting as desired. At the top of intake duct, pressure is decreasing due to the reason that duct is forward swept and directs the flow further away. So the phenomenon similar to expansion is occuring, and across expansion velocity increases and pressure is decreasing.

At supersonic Mach shock wave effects also come into play. Shock waves are generated at the bump apex and at the cowl lip of intake duct. Two shock waves must be intersecting in order to get shock on forward swept cowling lip. Multiple isentropic shock waves occur at bump surface which combine to generate a single stronger shock wave.

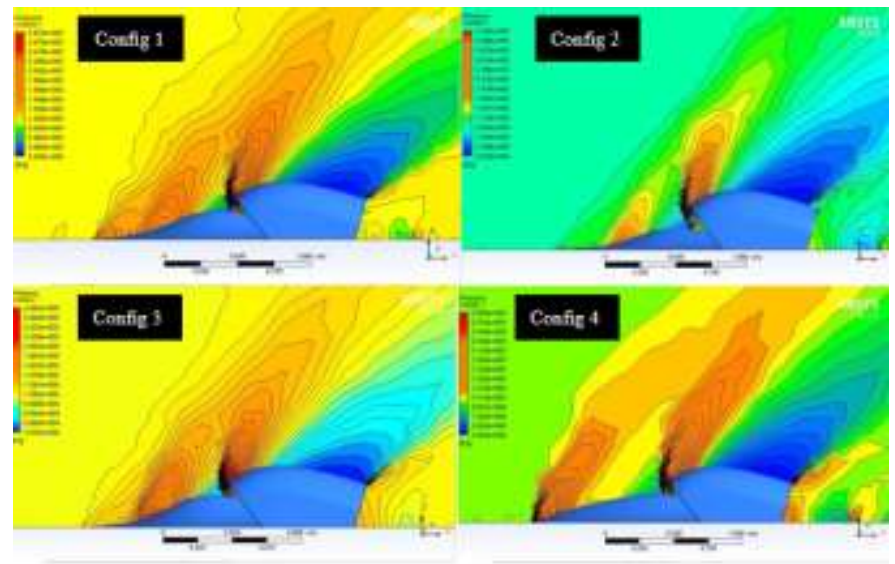

Fig. 9. Pressure Contours over bump configurations at Mach No. 1.5

Shock waves are clearly seen in the Fig 9. Config 4bump produces stronger shock wave due to its higher body angle. Also two shock waves (at bump apex and cowl lip) are not intersecting, so the shock on lip condition is not met. Shock waves are intersecting in case of Config 1, and associated turbulence is also less as compared to all other geometries, hence it results in better pressure recovery. Config 3 bump is also producing intersecting shocks but associated turbulence is much higher. Velocities downstream inlet duct are also calculated and it is observed that velocities are subsonic in all the cases except smaller bump. Config 2 bump is not able tochange the flow to subsonic speeds due to its smaller amplitude. Therefore, at supersonic speed higher amplitude of bump is preffered over smaller amplitude. In case of Config 1 bump, shock on lip phenomenon is met since its maximum amplitude is kept near the cowl lip. Pressure above the intake duct is almost same in all the cases since intake duct is same for all the cases so.

\section{Pressure Recovery}

Pressure recovery is the ratio of total pressure at engine interface and free stream total pressure. In this research total pressure downstream of intake duct is taken to be as total pressure at engine interface. A plane is made downstream of intake duct and pressure recovery is calculated by area wetted average for all four cases.

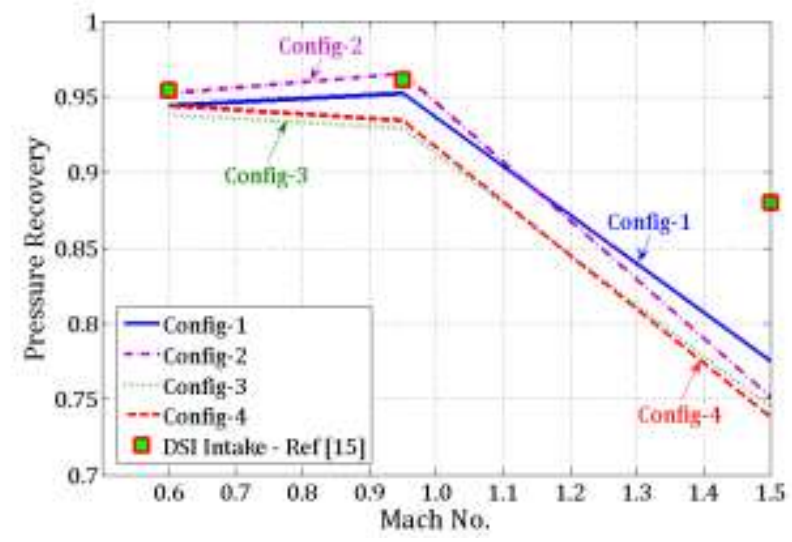

Fig. 10.Pressure Recovery at Engine Inlet for Various Bump Config

From Fig. 10 it is clearly evident that Config2 gives highest pressure recovery at Mach No 0.6. The difference in pressure recovery values increases as the Mach No. increases. At transonic and supersonic Mach No. the magnitude of pressure recovery becomes more pronounced. It is well established that a small difference in pressure recovery ahs major effect on intake efficiency[10]. The comparison between all four configurations shows that Config 1 and 2 have correspondingly higher pressure recoveries as compared to other configurations in all conditions. Config 1 shows higher pressure recovery at high supersonic speed, whereas Config 2 performance is better at subsonic, transonic and low supersonic speeds. Config 2 bump configuration provides lower pressure recovery at supersonic speed as compared to Config 1 configuration as it is unable to decelerate the flow to the required subsonic speed after passing from inlet duct. Pressure recovery of Config 3 and 4are slightly less than other bumps due to the reasons already discussed above in flow behavior analysis.

\section{Comparative Analysis with a Supersonic Fighter}

In order to examine the results and analyze the modeled bump geometries with an existing fighter aircraft having DSI configuration [5, 15], data points from Ref [15], corresponding to a fighter aircraft are shown in Figure 10. It can be seen that at subsonic speeds the pressure recovery is almost same for modeled bump and aircraft DSI configuration. But there is much difference at Mach No 0.95 between Config 3, Config 4 
and aircraft DSI configuration. At Mach 1.5, a prominent difference is observed between modeled bumps pressure recovery and aircraft configuration. This deviation is due to the fact that aircraft DSI configuration is optimally designed for supersonic speeds. This design includes bump geometry, positioning, forward swept angle of intake duct cowl lip and many other factors [10]. Hence, it is quite obvious that aircraft configuration is optimized and would yield highest pressure recovery than modeled bump geometries. Furthermore, the length of intake duct which was chosen for this analysis is not similar to actual length of aircraft duct which is subjected for comparison. Also, mass flow rate is not controlled in present analysis whereas pressure recovery at design mass flow rate is plotted for aircraft configuration. However, comparative analysis helped in verifying the variation of pressure recovery with the change in Mach No.

\section{CONCLUSION}

In this paper different bump surfaces were modeled and analyzed to evaluate their performance. Four 04 different bump configurations are modeled in MATALB $\AA$ based on their maximum amplitude, location of maximum amplitude, and initial / final bump angles. At subsonic and transonic speeds, Config 1 and 2 gave much similar pressure recovery to DSI configuration of aircraft, however, a significant difference in pressure recovery was observed at supersonic speeds. The deviation is due to the fact that aircraft DSI configuration is optimally designed for supersonic speeds.

\section{ACKNOWLEDGMENT}

The authors acknowledge the use of Numerical Analysis Lab (NAL) of College of Aeronautical Engineering, Risalpur, Pakistan.

\section{REFERENCES}

[1] Simon, P. C.; Brown, D. W.; and Huff, R. G. "Performance of external-compression bump inlet at Mach numbers of 1.5 and 2.0" NACA Report NACA-RM-E56L19 (1957).

[2] Sóbester, A., Tradeoffs in jet inlet design: A historical perspective. Journal of aircraft, 2007. 44(3): p. 705-717.

[3] Khan, O. and J. Masud, Evaluating an Experimental Streamlined Fairing for a Diverter Less Supersonic Inlet (DSI) Equipped Aircraft. AIAA.

[4] Masud, J. and F. Akram, Flow field and performance analysis of an integrated diverterless supersonic inlet. Aeronautical Journal, 2011. 115(1170): p. 471.

[5] Lim, S., Koh, D. H., Kim, S. D., and Song, D. J. “A Computational Study on the Efficiency of Boundary Layer Bleeding for the Supersonic Bump Type Inlet" AIAA-2009-34 47th AIAA Aerospace Sciences Meeting including The New Horizons Forum and Aerospace Exposition, Orlando, Florida, Jan. 5-8, 2009.

[6] Svensson, M., A CFD Investigation of a Generic Bump and its Application to a Diverterless Supersonic Inlet. 2008.

[7] Rasmussen, Maurice P., "Waverider configurations derived from inclined circular and elliptic cones." Journal of Spacecraft and Rockets 17.6 (1980): 537-545.

[8] Yang, Y. "Design of Bump Inlet of Thunder / JF-17 Aircraft" Journal of Nanjing University of Aeronautics \& Astronautics, Vol. 39, No. 4, August 2007, pp. 449-452.

[9] Kim, S. D. "Aerodynamic Design of a Supersonic Inlet with a Parametric Bump" Journal of Aircraft 2009 vol.46 no.1 (198-202).

[10] Seddon, J. and E.L. Goldsmith, Intake aerodynamics. 1999: Amer Inst of Aeronautics \& Astronautics.
[11] Liu, Yutong, et al. "The effects of different mesh generation methods on computational fluid dynamic analysis and power loss assessment in total cavopulmonary connection." Journal of biomechanical engineering 126.5 (2004): 594-603.

[12] FLUENT, Computational Fluid Dynamics Software Package User Guide.

[13] FLUENT, Computational Fluid Dynamics Software Package User Guide, Ver. 15.0, Fluent Inc, Lebanon, NH

[14] Spalarat, P., and Allmaras, S., "A One-Equation Turbulence Model for Aerodynamic Flows." Technical Report AIAA-92-0439, American Institute of Aeronautics and Astronautics, 1992

[15] Hassan, S., J. Masud, and O. Khan, Intake and Airframe Characterization through Composite CFD, 53rd AIAA Aerospace Sciences Meeting Kissimmee, Florida (2015).

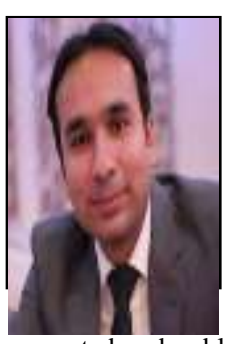

Engr. Irsalan Arif is an Assistant Professor at Department of Aerospace Engineering, College of Aeronautical Engineering, NUST. He completed his Bachelors Degree in Aerospace Engineering from College of Aeronautical Engineering, NUST and received President's Gold Medal in Aerospace Engineering. He later completed his Masters Degree in Fluid Dynamics for Air University. His has done substantial research in the field of Aerodynamics, External flows, Internal flows etc. He has already presented and published his work in AIAA Scitech 2017. He has a work experience of 05 years in aviation maintenance and 02 years in R\&D. 